\title{
Os cuidados de enfermagem no posicionamento anestésico-cirúrgico
}

\author{
Nursing care in anesthetic-surgical positioning \\ Atención de enfermería en el posicionamiento anestésico-quirúrgico
}

Recebido: 22/04/2021 | Revisado: 01/05/2021 | Aceito: 14/05/2021 | Publicado: 22/05/2021

\author{
Victor Hugo de Paula Flauzino \\ ORCID: https://orcid.org/0000-0001-5156-0030 \\ Faculdade Unyleya, Brasil \\ E-mail: prof.victorflauzino@unyleya.edu.br \\ Priscila Gramata da Silva Vitorino \\ ORCID: https://orcid.org/0000-0002-1201-6945 \\ Associação Brasileira de Enfermeiros Cientistas, Brasil \\ E-mail: prigramaenf@yahoo.com.br \\ Luana de Oliveira Hernandes \\ ORCID: https://orcid.org/0000-0001-8828-7146 \\ Associação Brasileira de Enfermeiros Cientistas, Brasil \\ E-mail: luanaoliveira2306@gmail.com \\ Daiana Moreira Gomes \\ ORCID: https://orcid.org/0000-0001-9387-0619 \\ Associação Brasileira de Enfermeiros Cientistas, Brasil \\ E-mail: daigomes_87@hotmail.com \\ Jonas Magno dos Santos Cesário \\ ORCID: https://orcid.org/0000-0003-1785-3555 \\ Faculdade Unyleya, Brasil \\ E-mail: prof.jonasmagno@unyleya.edu.br
}

\begin{abstract}
Resumo
Uma das preocupações da equipe de enfermagem se refere ao posicionamento do paciente durante a cirurgia, pois os agravos ocasionados pelo posicionamento anestésico-cirúrgico inadequado nas instituições de saúde representam um dos maiores comprometimento à saúde do paciente. $\mathrm{O}$ objetivo central do estudo foi descrever os principais cuidados de enfermagem durante o posicionamento anestésico-cirúrgico e mais especificamente, descrever os fatores de riscos para lesão no posicionamento anestésico-cirúrgico. Trata-se de uma revisão bibliográfica de abordagem descritiva e qualitativa, que utilizou as bases de dados do Google Acadêmico, BVS (biblioteca virtual em saúde) e da SciELO (Scientific Electronic Library Online). A pesquisa resultou em uma amostra final de 65 artigos, que permitiu compreender melhor o posicionamento cirúrgico adequado. Cabe ao enfermeiro todo o planejamento e implementação da assistência, bem como cuidados específicos destinados ao posicionamento anestésico-cirúrgico ideal. Observou-se que as ações do enfermeiro estão diretamente relacionadas ao gerenciamento da equipe, principalmente técnicos e auxiliares que irão manipular o paciente. Ao ser realizado o posicionamento do paciente na mesa cirúrgica, além de garantir a segurança do paciente, a equipe deverá se certificar que a área cirúrgica esteja bem exposta, com o corpo bem alinhado e que a pressão nos pontos de maior tensão possua proteção adequada. Foi possível observar uma escassez de publicações nacionais a respeito do assunto abordado neste estudo. Uma maior compreensão do tema contribui para mitigar os riscos relacionados ao posicionamento anestésico-cirúrgico.
\end{abstract}

Palavras-chave: Assistência perioperatória; Cuidados de enfermagem; Posicionamento do paciente.

\begin{abstract}
One of the concerns of the nursing team refers to the positioning of the patient during surgery, as the problems caused by inadequate anesthetic-surgical positioning in health institutions represent one of the greatest compromises to the patient's health. The main objective of the study was to describe the main nursing care during anesthetic-surgical positioning and more specifically, to describe the risk factors for injury in anesthetic-surgical positioning. It is a bibliographic review with a descriptive and qualitative approach, which used the databases of Google Scholar, VHL (virtual health library) and SciELO (Scientific Electronic Library Online). The research resulted in a final sample of 65 articles, which allowed a better understanding of the appropriate surgical positioning. It is up to the nurse to plan and implement care, as well as specific care for the ideal anesthetic-surgical position. It was observed that the nurse's actions are directly related to the management of the team, mainly technicians and assistants who will manipulate the patient. When positioning the patient on the operating table, in addition to ensuring patient safety, the team must make sure that the surgical area is well exposed, with the body well aligned and that the pressure at the highest tension points has adequate protection. It was possible to observe a shortage of national publications on the subject addressed
\end{abstract}


in this study. A greater understanding of the topic contributes to mitigate the risks related to anesthetic-surgical positioning.

Keywords: Perioperative care; Nursing care; Patient positioning.

\begin{abstract}
Resumen
Una de las preocupaciones del equipo de enfermería se refiere al posicionamiento del paciente durante la cirugía, ya que los problemas ocasionados por un posicionamiento anestésico-quirúrgico inadecuado en las instituciones de salud representan uno de los mayores compromisos para la salud del paciente. El objetivo principal del estudio fue describir los principales cuidados de enfermería durante el posicionamiento anestésico-quirúrgico y más específicamente, describir los factores de riesgo de lesión en el posicionamiento anestésico-quirúrgico. Se trata de una revisión bibliográfica con enfoque descriptivo y cualitativo, que utilizó las bases de datos de Google Scholar, VHL (biblioteca virtual de salud) y SciELO (Scientific Electronic Library Online). La investigación dio como resultado una muestra final de 65 artículos, lo que permitió una mejor comprensión del posicionamiento quirúrgico adecuado. Depende de la enfermera planificar e implementar los cuidados, así como los cuidados específicos para la posición ideal quirúrgicaanestésica. Se observó que las acciones de la enfermera están directamente relacionadas con la gestión del equipo, principalmente técnicos y asistentes que manipularán al paciente. Al colocar al paciente en la mesa de operaciones, además de garantizar la seguridad del paciente, el equipo debe asegurarse de que el área quirúrgica esté bien expuesta, con el cuerpo bien alineado y que la presión en los puntos de mayor tensión tenga la protección adecuada. Se pudo observar una escasez de publicaciones nacionales sobre el tema abordado en este estudio. Una mayor comprensión del tema contribuye a mitigar los riesgos relacionados con el posicionamiento anestésico-quirúrgico.
\end{abstract}

Palabras clave: Atención perioperatoria; Cuidado de enfermería; Posicionamiento del paciente.

\title{
1. Introdução
}

A segurança do paciente é indispensável para garantir o atendimento em todas as instituições de saúde, a área da saúde aprimora seus aspectos técnicos e científicos para gerar um aumento na quantidade e variedade de procedimentos cirúrgicos, os quais podem comprometer a segurança do paciente e impactar negativamente na recuperação no período pósoperatório. Uma das preocupações da equipe de enfermagem se refere ao posicionamento do paciente durante a cirurgia. Os agravos ocasionados pelo posicionamento anestésico-cirúrgico inadequado nas instituições de saúde representam um dos maiores comprometimento à saúde do paciente e é um grave problema de saúde pública (Guido et al., 2014).

O enfermeiro desempenha papel fundamental na implementação de estratégias junto à equipe de enfermagem, dentre elas a preocupação com a assistência de enfermagem é assegurar o posicionamento do cliente de forma adequada, por meio da prática baseada em evidências, o cotidiano do enfermeiro deve buscar o aperfeiçoamento no cuidado com a segurança, conforto e individualidade de cada paciente (Lopes et al., 2016).

$\mathrm{Na}$ atualidade, a maioria das organizações acreditadoras se empenham e estabelecem estratégias que assegurem a qualidade, segurança para minimizar e/ou eliminar situações prejudiciais no atendimento ao paciente, diante de inúmeros fatos que envolve a segurança do paciente se fez necessário que o Ministério da Saúde, por meio da portaria 529, de $1^{\circ}$ de abril de 2013, normatizasse o Programa Nacional de Segurança do Paciente (PNSP), para incentivar a inclusão da temática ligada à segurança do paciente na formação acadêmica dos profissionais da área da saúde (Mendes, Araújo \& Morgan, 2020).

A Sistematização da Assistência em Enfermagem Perioperatório (SAEP), é um processo utilizado no centro cirúrgico gerenciada pelo enfermeiro e executada por sua equipe. Este planejamento individual zela pela segurança do cliente submetido a procedimentos anestésico-cirúrgicos e garante a qualidade da assistência (Silva et al., 2015).

Para o sucesso da assistência de enfermagem é necessário que o enfermeiro possua conhecimentos científicos relacionados ao tempo cirúrgico, a exposição e tipos de anestésicos que podem ser utilizados, material para o posicionamento adequado, doenças de base, fármacos em uso, antecedentes alérgicos e todas as alterações fisiológicas que comprometam a segurança do paciente no período intraoperatório (Ribeiro, Peniche \& Silva, 2017).

Os fatores de riscos relacionados à lesão no posicionamento anestésico-cirúrgico são divididos em: intrínsecos, extrínsecos e superfície corporal. Para prevenir as lesões de pele durante o posicionamento deve ser utilizado os seguintes dispositivos: colchões de polímero e protetores de proeminências ósseas. Os fatores extrínsecos são relacionados a umidade 
exagerada da pele (ocasionada pela antissepsia), fricção, cisalhamento, aquecimento, tempo anestésico-cirúrgico, agentes anestésicos, alterações hemodinâmicas, posição escolhida, uso de mesa e colchão inapropriados, além de ambiente com temperatura inadequada (Trevilat et al., 2018).

A não realização do adequado posicionamento anestésico-cirúrgico pode levar a alterações fisiológicas e afetar alguns sistemas como: sistema nervoso, respiratório, cardiovascular, músculo esquelético, tegumentar (equimose, úlcera por pressão, eritema, queimaduras) e até lesões articulares (estiramento, luxações, esforço musculares). A hipotermia gerada pela perda de barreiras protetoras, assim como o ambiente frio e úmido que o cliente está inserido, também favorecem o surgimento de lesões. Podem ocorrer queimaduras por placas dispositivas de eletro cautério, utilizadas incorretamente e até mesmo instalações elétricas inapropriadas podem comprometer a qualidade da assistência de enfermagem (Oliveira et al., 2019)

O posicionamento do paciente para o recebimento da anestesia é um procedimento peculiar realizado pela equipe multiprofissional e pode interferir em todas as fases do período perioperatório. O treinamento e a supervisão dos enfermeiros realizados passam a assegurar e direcionar as realizações, através dos protocolos que junto às necessidades identificadas, desenvolvem e implementam uma assistência sistematizada e segura para o paciente. O paciente exposto a um período longo de procedimento anestésico-cirúrgico apresenta riscos devido a vasodilatação periférica, diminuição do retorno venoso ao coração e pulmões, perda da capacidade sensitiva de uma pequena parte ou de todo corpo, incapacidade de reação à dor e lesão tecidual ocasionada por pressão e compressão de tecidos, lesão tecidual. Diante deste problema, os autores buscaram identificar quais são os cuidados de enfermagem durante o posicionamento anestésico-cirúrgico. O objetivo central do estudo foi descrever os principais cuidados de enfermagem durante o posicionamento anestésico-cirúrgico e mais especificamente, descrever os fatores de riscos para lesão no posicionamento anestésico-cirúrgico.

\section{Metodologia}

Este estudo é uma revisão bibliográfica de abordagem descritiva e qualitativa, descrita por Cesário, Flauzino e Mejia (2020) como um tipo de revisão fundamentada na literatura, o que incluí artigos científicos publicados em periódicos acadêmicos. No DeCS (Descritores em Ciências da Saúde), encontrou-se os seguintes descritores: Assistência perioperatória, Cuidados de enfermagem e Posicionamento do paciente. Os bancos de dados utilizados foram o Google Acadêmico, BVS (biblioteca virtual em saúde) e SciELO (Scientific Electronic Library Online). No Google Acadêmico utilizou-se cada um dos descritores entre aspas ("'), já na BVS, foi utilizado a opção pesquisa avançada, selecionada as bases da BDENF (Banco de Dados em Enfermagem), LILACS (Literatura Latino-Americana e do Caribe em Ciências da Saúde) e o operador lógico booleano "OR" e "AND". Por fim, na SciELO, foi utilizada a opção pesquisa avançada e o operador lógico booleano "OR" e "AND".

Estabeleceu-se como critérios de inclusão, artigos acadêmicos publicados entre 2014 e 2021, na língua portuguesa, disponíveis de forma gratuita e nos bancos de dados já mencionados, que respondessem à pergunta de pesquisa. Excluíram-se artigos repetidos encontrados nas bases de dados, resumos, artigos inferiores a 2014 e artigos que não respondiam o problema da pesquisa. A coleta dos dados foi realizada no mês de março, por dois pesquisadores de forma independente. Os resultados das buscas pelos dados e do número final de publicações que compõem a revisão foram apresentados por meio do fluxograma de Prisma (Peters, 2015), conforme a Figura 1:

Figura 1. Fluxograma de Prisma. 


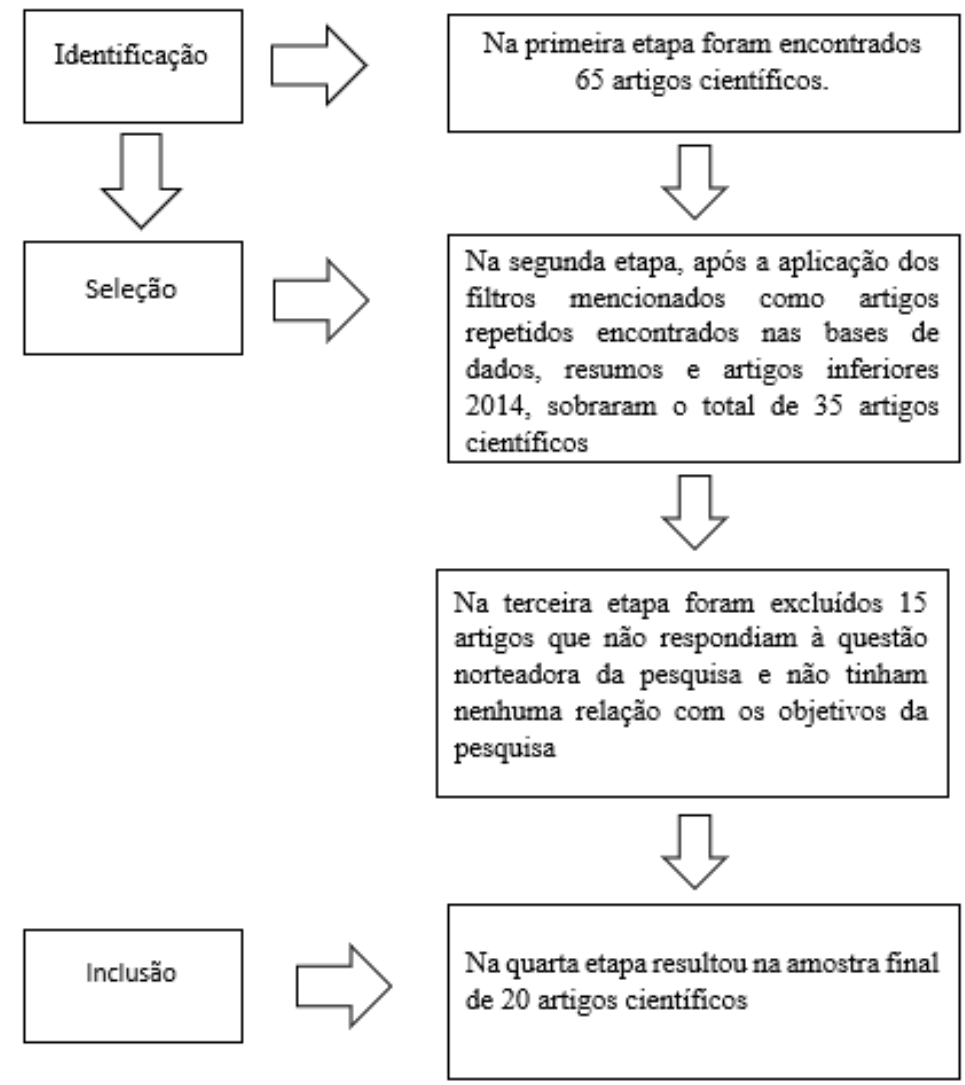

Fonte: Autores (2021).

\section{Resultados}

A Tabela 1 mostra os artigos científicos incluídos em cada base de dados, e possível observa que o maior aproveitamento de artigos foi da BVS, seguida pelo Google Acadêmico e, por fim, SciELO.

Tabela 1. Resultados da busca nas bases de dados.

\begin{tabular}{l|c|c}
\multicolumn{1}{c}{ Base de dados } & Total & Ancluídos \\
\cline { 2 - 3 } \multicolumn{1}{c}{ BVS } & 30 & 9 \\
\hline Google Scholar & 25 & 6 \\
\hline SciELO & 10 & 5 \\
\hline
\end{tabular}

Fonte: Autores (2021).

Para apresentar um melhor entendimento das publicações, foi elaborado o Quadro 1, que mostra uma síntese dos artigos selecionados com as seguintes: autor, título, objetivo central e tipo de estudo. Antes da discussão do trabalho ser realizada, os estudos foram organizados no Quadro 1, logo abaixo, que contribuiu para comparar os assuntos que foram abordados nesta pesquisa.

Quadro 1. Artigos incluídos neste estudo. 
Research, Society and Development, v. 10, n. 6, e34410615358, 2021

(CC BY 4.0) | ISSN 2525-3409 | DOI: http://dx.doi.org/10.33448/rsd-v10i6.15358

\begin{tabular}{|c|c|c|c|}
\hline Autor/ano & Título & Objetivo do Central & Tipo de estudo \\
\hline $\begin{array}{l}\text { Guido et al., } \\
2014\end{array}$ & $\begin{array}{lr}\text { Cuidado de } & \text { enfermagem } \\
\text { perioperatório: } & \text { revisão } \\
\text { integrativa de literatura. }\end{array}$ & $\begin{array}{l}\text { conhecer quais são os cuidados de enfermagem } \\
\text { realizados no período perioperatório }\end{array}$ & $\begin{array}{l}\text { Revisão integrativa de } \\
\text { literatura. }\end{array}$ \\
\hline Silva et al., 2015 & $\begin{array}{l}\text { Análise de eventos adversos } \\
\text { em um centro cirúrgico } \\
\text { ambulatorial }\end{array}$ & $\begin{array}{l}\text { Analisar os eventos adversos notificados no Centro } \\
\text { Cirúrgico para a segurança do paciente }\end{array}$ & $\begin{array}{l}\text { Estudo documental } \\
\text { retrospectivo }\end{array}$ \\
\hline $\begin{array}{l}\text { Lopes et al., } \\
2016\end{array}$ & $\begin{array}{l}\text { Construir e validar escala de } \\
\text { avaliação de risco para lesões } \\
\text { decorrentes do posicionamento } \\
\text { cirúrgico em pacientes adultos. }\end{array}$ & $\begin{array}{l}\text { construir e validar escala de avaliação de risco para } \\
\text { lesões decorrentes do posicionamento } \\
\text { cirúrgico em pacientes adultos. }\end{array}$ & $\begin{array}{l}\text { Pesquisa descritiva, } \\
\text { retrospectiva, } \\
\text { abordagem quantitativa }\end{array}$ \\
\hline $\begin{array}{l}\text { Miranda et al., } \\
2016\end{array}$ & $\begin{array}{l}\text { Posicionamento cirúrgico: } \\
\text { cuidados de enfermagem no } \\
\text { transoperatório. }\end{array}$ & $\begin{array}{l}\text { Identificar os cuidados de enfermagem no } \\
\text { posicionamento, relatando as complicações }\end{array}$ & $\begin{array}{l}\text { Revisão integrativa da } \\
\text { literatura }\end{array}$ \\
\hline $\begin{array}{l}\text { Angelo et al., } \\
2017\end{array}$ & $\begin{array}{l}\text { Efetividade do protocolo } \\
\text { prevenção de lesões de pele } \\
\text { em cirurgias urológicas } \\
\text { robóticas }\end{array}$ & $\begin{array}{l}\text { Verificar a efetividade do Protocolo Prevenção de } \\
\text { Lesão de Pele, por meio do levantamento de } \\
\text { ocorrências causadas pelo posicionamento cirúrgico } \\
\text { em pacientes oncológicos submetidos às cirurgias } \\
\text { urológicas robóticas e demonstrar a importância da } \\
\text { simulação como estratégia educativa no } \\
\text { treinamento da equipe de enfermagem }\end{array}$ & $\begin{array}{l}\text { Pesquisa descritiva, } \\
\text { retrospectiva, } \\
\text { abordagem quantitativa }\end{array}$ \\
\hline $\begin{array}{l}\text { Ribeiro, Peniche } \\
\text { \& Silva, } 2017\end{array}$ & $\begin{array}{l}\text { Complicações na sala de } \\
\text { recuperação anestésica, fatores } \\
\text { de riscos e intervenções de } \\
\text { enfermagem: } \\
\text { integrativa }\end{array}$ & $\begin{array}{l}\text { Identificar as complicações e os riscos que os } \\
\text { pacientes possam desenvolver no pós-operatório } \\
\text { imediato e propor um instrumento que contenha as } \\
\text { complicações, riscos e intervenções de enfermagem } \\
\text { individualizada. }\end{array}$ & Revisão integrativa \\
\hline $\begin{array}{l}\text { Araújo, Silva \& } \\
\text { Pereira, } 2018\end{array}$ & $\begin{array}{l}\text { Lesão por pressão no } \\
\text { transoperatório de cirurgia } \\
\text { cardíaca. }\end{array}$ & $\begin{array}{l}\text { Identificar as intervenções de enfermagem eficazes } \\
\text { para formas de prevenção de lesão por pressão } \\
\text { (LPP) a pacientes submetidos à cirurgia cardíaca. }\end{array}$ & Revisão de literatura \\
\hline $\begin{array}{l}\text { Gomes et al., } \\
2018\end{array}$ & $\begin{array}{l}\text { Lesão por abrasão após } \\
\text { cateterismo cardíaco: relato de } \\
\text { caso }\end{array}$ & $\begin{array}{l}\text { Relatar um caso de lesão de pele por abrasão } \\
\text { relacionada à retirada de curativo pós-cateterismo } \\
\text { cardíaco via artéria femoral }\end{array}$ & Relato de um caso \\
\hline $\begin{array}{l}\text { Loiola et al., } \\
2018\end{array}$ & $\begin{array}{l}\text { Avaliação de risco no } \\
\text { posicionamento cirúrgico: } \\
\text { relato de experiência }\end{array}$ & $\begin{array}{l}\text { Relatar a experiência de implantação da escala de } \\
\text { posicionamento cirúrgico em um centro cirúrgico } \\
\text { de um hospital particular, no protocolo de } \\
\text { prevenção de lesão por pressão em decorrência do } \\
\text { posicionamento cirúrgico }\end{array}$ & $\begin{array}{l}\text { Estudo descritivo, } \\
\text { quantitativo, do tipo } \\
\text { relato de experiência }\end{array}$ \\
\hline Sousa, Bispo \& & Criação de um manual para & Relatar a experiência da criação de um manual de & Relato de experiência \\
\hline
\end{tabular}




\begin{tabular}{|c|c|c|c|}
\hline Acunã, 2018 & $\begin{array}{l}\text { posicionamento cirúrgico: } \\
\text { relato de experiência. }\end{array}$ & posicionamento cirúrgico. & \\
\hline $\begin{array}{l}\text { Trevilato, } \\
\text { Fagundes \& } \\
\text { Caregnato, } 2018\end{array}$ & $\begin{array}{l}\text { Posicionamento cirúrgico: } \\
\text { Prevalência de risco de lesões } \\
\text { em pacientes cirúrgicos }\end{array}$ & $\begin{array}{l}\text { Determinar a prevalência de pacientes em risco de } \\
\text { desenvolvimento de lesões decorrentes do } \\
\text { posicionamento cirúrgico. }\end{array}$ & Estudo transversal \\
\hline $\begin{array}{l}\text { Bezerra et al., } \\
2019\end{array}$ & $\begin{array}{l}\text { Fatores associados a lesões de } \\
\text { pele decorrentes do período } \\
\text { intraoperatório. }\end{array}$ & $\begin{array}{l}\text { Avaliar a ocorrência de lesões de pele no período } \\
\text { intraoperatório decorrentes de procedimentos } \\
\text { cirúrgicos realizados em um hospital universitário } \\
\text { de grande porte. }\end{array}$ & $\begin{array}{l}\text { Estudo transversal, } \\
\text { descritivo, com } \\
\text { abordagem quantitativa }\end{array}$ \\
\hline $\begin{array}{l}\text { Oliveira et al., } \\
2019\end{array}$ & $\begin{array}{l}\text { Avaliação do risco para o } \\
\text { desenvolvimento de lesões } \\
\text { perioperatórias decorrentes do } \\
\text { posicionamento cirúrgico }\end{array}$ & $\begin{array}{l}\text { Avaliar o risco de desenvolvimento de lesões } \\
\text { perioperatórias decorrentes do posicionamento } \\
\text { cirúrgico em pacientes submetidos a cirurgias } \\
\text { eletivas. }\end{array}$ & $\begin{array}{l}\text { Estudo analítico e } \\
\text { longitudinal }\end{array}$ \\
\hline $\begin{array}{l}\text { Peixoto } \\
\text { et al., } 2019\end{array}$ & $\begin{array}{l}\text { Classificação de risco de } \\
\text { desenvolvimento de lesões } \\
\text { decorrentes do posicionamento } \\
\text { cirúrgico }\end{array}$ & $\begin{array}{l}\text { Avaliar e classificar pacientes segundo a Escala de } \\
\text { Avaliação de Risco para Desenvolvimento de } \\
\text { Lesões Decorrentes do Posicionamento Cirúrgico. }\end{array}$ & $\begin{array}{l}\text { Estudo observacional, } \\
\text { longitudinal, } \\
\text { prospectivo e } \\
\text { quantitativo }\end{array}$ \\
\hline Pinto et al., 2018 & $\begin{array}{l}\text { Posicionamento do paciente } \\
\text { para raquianestesia: construção } \\
\text { e validação de álbum seriado }\end{array}$ & $\begin{array}{l}\text { Construir e validar álbum seriado educativo para } \\
\text { gestantes que serão submetidas à cirurgia cesariana, } \\
\text { acerca do posicionamento durante a raquianestesia. }\end{array}$ & $\begin{array}{l}\text { Estudo metodológico } \\
\text { realizado com a } \\
\text { elaboração }\end{array}$ \\
\hline $\begin{array}{l}\text { Sandes et al., } \\
2019\end{array}$ & $\begin{array}{l}\text { Lesões provenientes de } \\
\text { procedimento cirúrgico: } \\
\text { fatores relacionados. }\end{array}$ & $\begin{array}{l}\text { Analisar as publicações científicas referentes a } \\
\text { lesões de pele decorrentes de procedimento } \\
\text { cirúrgico e identificar os fatores de risco associados } \\
\text { à ocorrência das lesões }\end{array}$ & $\begin{array}{l}\text { Estudo de revisão } \\
\text { integrativa de literatura }\end{array}$ \\
\hline $\begin{array}{l}\text { Ângelo et al., } \\
2020\end{array}$ & $\begin{array}{l}\text { Posicionamento cirúrgico em } \\
\text { cirurgia robótica pediátrica: } \\
\text { relato de experiência }\end{array}$ & $\begin{array}{l}\text { Relatar a experiência dos primeiros seis casos de } \\
\text { cirurgia robótica pediátrica e a atuação dos } \\
\text { enfermeiros especialistas em cirurgia robótica em } \\
\text { uma instituição oncológica }\end{array}$ & Relato de experiência \\
\hline $\begin{array}{l}\text { Mendes, Araújo } \\
\text { \& Morgan, } 2020\end{array}$ & $\begin{array}{l}\text { Atuação do enfermeiro na } \\
\text { prevenção de eventos adversos } \\
\text { no centro cirúrgico, utilizando } \\
\text { SAEP }\end{array}$ & $\begin{array}{l}\text { Escrever a assistência do enfermeiro na prevenção } \\
\text { dos eventos adversos, compreendendo as falhas } \\
\text { sistêmicas }\end{array}$ & Revisão de literatura \\
\hline $\begin{array}{l}\text { Macedo \& } \\
\text { Vasconcelos, } \\
2021\end{array}$ & $\begin{array}{l}\text { O uso seguro da eletrocirurgia } \\
\text { no período intraoperatório: } \\
\text { evidências para o cuidado de } \\
\text { enfermagem }\end{array}$ & $\begin{array}{l}\text { Analisar a produção científica publicada a respeito } \\
\text { do cuidado de enfermagem frente ao uso da } \\
\text { eletrocirurgia }\end{array}$ & Revisão integrativa \\
\hline
\end{tabular}

\section{Discussão}

Fonte: Autores (2021). 
Com o avanço científico e tecnológico na área hospitalar, com instrumentais cada vez mais sofisticados e técnicas cirúrgicas menos invasivas, mas ainda sim de alta complexidade, faz-se necessário uma fundamentação científica para garantir um modelo assistencial ideal ao paciente. Esse modelo, para a enfermagem perioperatória, é denominado SAEP (Sistema de Assistência de Enfermagem Perioperatório), que contribuir para padronizar ações e garantir a qualidade da assistência. A SAEP contempla uma assistência individualizada, integral, contínua, participativa, documentada e avaliada continuamente, com uma assistência ligada as intervenções, promove a continuidade dos cuidados, inserção familiar com o propósito de avaliação e satisfação na assistência (Angelo et al., 2017).

A SAEP é um processo que apresenta objetivos específicos como promover, manter e recuperar a saúde do cliente. Essa sistematização se inicia no período pré-operatório imediato, continua no transoperatório e se estende até o pós-operatório imediato. Dessa forma é possível que haja uma maior interação entre paciente e enfermeiro, que tem a oportunidade de conhecer os possíveis problemas e planejar individualmente as ações para prevenir complicações durante toda a assistência de enfermagem no período perioperatório (Mendes, Araújo \& Morgan, 2020).

O gerenciamento da SAEP é privativo do enfermeiro, que deverá identificar e analisar as necessidades do paciente, como objetivo de minimizar os riscos de complicações no período perioperatório. A sistematização é fundamental para nortear as ações e cuidados executados ao paciente cirúrgico, dividida em cinco etapas: visita pré-operatória, diagnósticos de enfermagem, prescrição de enfermagem, implementação e visita no pós-operatório (Miranda et al., 2016).

Para um cuidado de qualidade durante todo o processo cirúrgico é necessária uma equipe organizada e consciente de suas ações para ofertar o melhor cuidado ao paciente cirúrgico, por meio do uso de conhecimento científico atualizado, habilidades e destreza técnicas. O enfermeiro possui um papel fundamental na equipe durante a visita pré-operatória e em todo planejamento do transoperatório e pós-operatório (Oliveira et al., 2019).

O enfermeiro deve realizar as atividades em conjunto com a equipe para que juntos seja possível uma avaliação constante da forma de atuação, assim como corrigir eventuais falhas no atendimento. Além disso, conhecer o cliente é essencial para executar um plano seguro, adequado e organizado, que atenda às suas necessidades (Loiola et al., 2018).

O cliente fica exposto a diversas complicações, pela perda temporária da função cognitiva, assim como uma capacidade de percepção dolorosa prejudicada, por isso a equipe de enfermagem deve planejar o cuidado para recuperar o equilíbrio fisiológico do cliente e prevenir o máximo de complicações possíveis. Individualizar o cuidado e identificar corretamente as necessidades do paciente irá contribuir para uma assistência segura (Peixoto et al., 2019).

O enfermeiro possui a responsabilidade de oferecer um ambiente calmo, seguro, limpo e confortável para a segurança do paciente, assim como promover ações de prevenção de injúrias e provisão de equipamentos e materiais que serão utilizados no ato anestésico-cirúrgico. Os fatores de risco para lesão devem ser considerados com o intuito de apresentar um plano assistencial que contribua diretamente na prevenção de complicações provenientes do período intraoperatório. (Sousa, Bispo \& Acunã, 2018).

O paciente submetido à anestesia perde sua capacidade sensitiva de partes ou de todo o corpo, não reage a dor, também há perda da capacidade de movimentação e dessa forma, se o cliente for mantido em posições inadequadas, o risco de lesão será elevado. O posicionamento provém de três posições básicas que são: decúbito dorsal (SUPINA), decúbito ventral (PRONA) decúbito lateral (SIMS) (Guido et al., 2014). Como o uso de anestésico causa um efeito relaxante para os músculos, as lesões ocasionadas no intraoperatório podem ocorrer com maior facilidade, portanto para minimizar os riscos de lesão, ao posicionar o cliente devem ser considerados os fatores de risco e evitar pressão e fricção sob a pele do paciente, com medidas específicas em cada uma das posições básicas (Bezerra et al., 2020).

Ao posicionar o paciente portador de DPOC (doença pulmonar obstrutiva crônica) é importante favorecer um padrão respiratório eficaz, que proporcione o aporte adequado de oxigênio. Um posicionamento inadequado pode levar a uma 
diminuição no tempo de ventilação entre a inspiração e expiração de forma desproporcional, diminuição da frequência respiratória e a queda de saturação de oxigênio. Os tecidos mais profundos desenvolvem hipóxia em função do tempo de compressão exercida pelo posicionamento, além de aumentar o risco de aparecimento de úlcera por pressão (Sandes et al., 2019).

Os pacientes emagrecidos possuem uma diminuição de massa muscular e tecido adiposo, menor espessura da derme e epiderme, o que torna esses indivíduos mais suscetíveis para desenvolver infecção e úlcera por pressão, já que também apresentam um sistema imunológico debilitado. Existe exposição acentuada de proeminência óssea, o que aumenta o risco de lesão por posicionamento inadequado durante procedimentos anestésicos cirúrgicos, dessa forma o enfermeiro deve garantir a correta avaliação deste local e adotar medidas para reduzir o friccionamento do tecido junto ao colchão (Ribeiro, Peniche \& Silva, 2017).

Os pacientes nos extremos de idade apresentam uma fragilidade no tecido tegumentar, diminuição de glândulas sudoríparas e sebáceas, que deixam a pele mais seca, com menor elasticidade, flácida e com diminuição do tecido epitelial, fisiologicamente mais frágil. Os idosos tendem a ter um desequilíbrio nutricional o que interfere diretamente na recuperação pós-operatória, o que aumenta o risco de lesão de pele e infecção (Trevilato, Fagundes \& Caregnato, 2018).

A hipotermia (temperatura abaixo de $36^{\circ} \mathrm{C}$ ) a que o paciente é exposto na sala cirúrgica diminui o seu metabolismo. A utilização de drogas vasodilatadoras, relaxantes, analgésicas e hipnóticas, possuem ação no centro termorregulador da temperatura, que por sua vez deprime o hipotálamo e impede que o organismo reaja à baixa temperatura. Esses eventos contribuem para a diminuição da contração muscular e com a perda de calor, sem o aporte de oxigênio adequado, pode acarretar hipóxia e favorecer a formação de necrose tecidual. A infusão de volume intravenoso aquecido, como o soro fisiológico $0,9 \%$, ringer simples ou ringer lactato, assim como o uso de manta e colchão térmico reduzem o risco de lesão de pele no momento intraoperatório (Bezerra et al., 2019).

A imobilidade dificulta o posicionamento cirúrgico e isso pode levar a formação de pontos de pressão, pois diminui o fluxo sanguíneo no local. Existe a possibilidade de ocorrer uma limitação da expansão pulmonar pela pressão da posição exercida sobre os arcos costais. Ao chegar em um valor de pressão de $32 \mathrm{mmHg}$, os micro vasos colapsam, o que favorece a formação de trombos, limita o fluxo sanguíneo tecidual e leva a privação na quantidade de oxigênio recebida. Sem oxigenação e outros nutrientes essenciais para atender às necessidades do organismo, ocorre a produção de metabolitos tóxicos nos tecidos. Com o extravasamento de líquido para o espaço intersticial, ocorre a formação de edema e isso tem o potencial de causar a morte celular do tecido. Com a morte do tecido, há um maior risco para o aparecimento de úlcera por pressão (Ângelo et al., 2020).

Em um tempo cirúrgico prolongado, com mais de 2 horas de duração, somado aos efeitos de drogas anestésicas, contribuem para aumento do risco de lesão tecidual, relacionada a isquemia tissular (Lopes et al., 2016). Esse risco não deve ser negligenciado pela equipe de enfermagem, que deve tomar medidas para garantir a segurança do paciente no momento do posicionamento anestésico-cirúrgico. A necessidade de submeter o cliente a várias posições na mesa cirúrgica, ou ainda manter em uma mesma posição, requer precisão, destreza e competência da equipe multidisciplinar na manipulação do paciente. para realizar de forma correta o posicionamento e garantir a segurança do cliente com o objetivo de prevenir complicações.

As posições básicas do paciente na mesa cirúrgica variam conforme o procedimento cirúrgico e qual sistema orgânico será abordado pela equipe, o que exige conhecimento avançado de anatomia, fisiologia e patologias. O posicionamento adequado é aquele que proporciona uma boa exposição da área cirúrgica, mantém o alinhamento corporal, minimiza a tensão e pressão exercidas sobre nervos e músculos. Dessa maneira, a escolha do posicionamento depende de diferentes fatores, o que inclui o tipo de procedimento, a localização do sítio operatório e a preferência do cirurgião, desde que seja possível garantir a segurança do paciente (Gomes et al., 2018). 


\section{Considerações Finais}

Cabe ao enfermeiro todo o planejamento e implementação da assistência, bem como cuidados específicos destinados ao posicionamento anestésico-cirúrgico ideal. O planejamento deve considerar as necessidades dos pacientes e ser elaborado de maneira individualizada. $\mathrm{O}$ atendimento sistematizado garante a continuidade da assistência e contribui para evitar falhas por membros da equipe. Foi possível observar que as ações do enfermeiro estão diretamente relacionadas ao gerenciamento da equipe, principalmente técnicos e auxiliares que irão manipular o paciente. Ao ser realizado o posicionamento do paciente na mesa cirúrgica, além de garantir a segurança do paciente, a equipe deverá se certificar que a área cirúrgica esteja bem exposta, com o corpo bem alinhado e que a pressão nos pontos de maior tensão possua proteção adequada. Para isso, é importante que a tomada de decisão do enfermeiro seja construída por meio de conceitos e práticas baseadas em evidências, com a elaboração de protocolos de posicionamento que levem em consideração as particularidades de cada paciente e às necessidades de cada posição. O treinamento e supervisão constantes do enfermeiro são necessárias para garantir a qualidade do atendimento da equipe de enfermagem e mitigar os riscos relacionados ao posicionamento na mesa cirúrgica.

Foi possível observar uma escassez de publicações nacionais a respeito do assunto abordado neste estudo. Dessa forma, os autores esperam que este estudo contribua para novas pesquisas relacionadas as técnicas de posicionamento anestésico-cirúrgico.

\section{Referências}

Angelo, C. S et al. (2017). Efetividade do protocolo prevenção de lesões de pele em cirurgias urológicas robóticas. Revista SOBECC; 22(3). 152-160.

Ângelo, C. S et al. (2020). Posicionamento cirúrgico em cirurgia robótica pediátrica: relato de experiência. Revista SOBECC. 25(2): 120-123

Araújo, L. C. N., Silva, F. M. \& Pereira, V. A. (2018). Lesão por pressão no transoperatório de cirurgia cardíaca. GEP NEWS. 1(1). 8-13.

Bezerra, M. B. G et al. (2019). Fatores associados a lesões de pele decorrentes do período intraoperatório. Revista SOBECC; 24(2). 76-84.

Bezerra, S. M. G et al. (2020). Estratégias de enfermagem para prevenção de lesão por pressão em pacientes cirúrgicos Brazilian Journal of Enterostomal Therapy. 18. e1020.

Cesário, J. M. S., Flauzino, V. H. P. \& Mejia, J. V. C. (2020). Metodologia científica: Principais tipos de pesquisas e suas caraterísticas. Revista Científica Multidisciplinar Núcleo do Conhecimento. 5(11). 23-33.

Gomes, E. T et al. (2018) Lesão por abrasão após cateterismo cardíaco: relato de caso. Revista SOBECC. 23(2). 109-113.

Guido, L. A et al. (2014). Cuidado de enfermagem perioperatório: revisão integrativa de literatura. Revista de pesquisa cuidado fundamental online. 6(4).1601-1609

Loiola, H. A. B et al. (2018). Avaliação de risco no posicionamento cirúrgico: relato de experiência. Revista de Enfermagem UFPI. 7(2):86-9.

Lopes, C. M. M et al. (2016). Escala de avaliação de risco para lesões decorrentes do posicionamento cirúrgico. Revista Latina Americana de Enfermagem. 24. e2704.

Macedo, K. S. S. \& Vasconcelos, E. L. (2021). O uso seguro da eletrocirurgia no período intraoperatório: evidências para o cuidado de enfermagem. Research, Society and Development. 10(1). e1210111203.

Mendes, P. J. A., Araújo, K. C. G. S., \& Morgan, P. E. M. (2020). Atuação do enfermeiro na prevenção de eventos adversos no centro cirúrgico, utilizando SAEP. Editorial BIUS, 19(13).

Miranda, A. B et al. (2016). Posicionamento cirúrgico: cuidados de enfermagem no transoperatório. Revista SOBECC. 21(1). 52-58.

Oliveira, H. M. B. S et al. (2019). Avaliação do risco para o desenvolvimento de lesões perioperatórias decorrentes do posicionamento cirúrgico. Revista Gaúcha de Enfermagem. 40. e20180114.

Peixoto, C. A et al. (2019). Classificação de risco de desenvolvimento de lesões decorrentes do posicionamento cirúrgico. Revista Latino-Americana de Enfermagem. 27. e3117.

Peters, M. D et al. (2015). Guidance for conducting systematic scoping reviews. International Journal of Evidence-Based Healthcare. 13(3). $141-146$.

Pinto, S. L et al. (2018). Posicionamento do paciente para raquianestesia: construção e validação de álbum seriado. Acta Paul Enfermagem. 31 (1). 25-31. 
Research, Society and Development, v. 10, n. 6, e34410615358, 2021

(CC BY 4.0) | ISSN 2525-3409 | DOI: http://dx.doi.org/10.33448/rsd-v10i6.15358

Sandes, S. M. S et al. (2019). Lesões provenientes de procedimento cirúrgico: fatores relacionados Revista SOBECC. 24(3). 161-167.

Silva, F. G. S. et al. (2015). Análise de eventos adversos em um centro cirúrgico ambulatorial. Revista SOBECC. 20(4). 202-209

Sousa, C. S., Bispo, D. M. \& Acunã, A. A. (2018;). Criação de um manual para posicionamento cirúrgico: relato de experiência. Revista SOBECC. 23(3). 169175.

Ribeiro, M. B., Peniche, A. C. G., \& Silva S. C. F. (2017) Complicações na sala de recuperação anestésica, fatores de riscos e intervenções de enfermagem: Revisão integrativa. Revista SOBECC. 22(4). 218-229

Trevilato, D. D et al. (2018). Posicionamento cirúrgico: Prevalência de risco de lesões em pacientes cirúrgicos. Revista SOBECC, 23(3): 124-129. 